\title{
Evaluation of Vehicle Tracking for Traffic Monitoring Based on Road Surface Mounted Magnetic Sensors
}

\author{
Ali M. H. Kadhim*, Wolfgang Birk and Thomas Gustafsson \\ Control Engineering Group at Luleå University of Technology, Luleå, \\ Sweden (e-mail:\{ali.kadhim,wolfgang.birk, tgu\}@ltu.se). \\ ${ }^{*}$ Corresponding author.
}

\begin{abstract}
The aim of this work is to evaluate a vehicle tracking scheme as a means of monitoring traffic on roads. The scheme can be used as a component in a traffic monitoring system which can provide traffic management systems and road maintainers with traffic information. Vehicle tracking is achieved by determining vehicle position, velocity and magnetic moment using a nonlinear weighted least squares method $(N W L S)$ on readings from two 3 -axes magnetic sensors. The tracking was performed both in simulation and in real life. The traffic monitoring system is composed of two adjacently glue attached wireless sensor nodes, which are placed at a distance of $1 \mathrm{~m}$ along the road. A potential misalignment of the sensors due to placement errors is analyzed in simulation and addressed.
\end{abstract}

\section{INTRODUCTION}

Fatality in road traffic is the dominating cause for nonnatural human death in the European society. Although there are many efforts to equip vehicles in order to avoid accident by alerting the driver or by taking the action themselves, see Eidehall [2007], such techniques have not reached all road users yet.

Another technique, proposed by Birk [2009], is to glueattach intelligent road markings onto the road surface. These markings, called Road Markings Units (RMU), consist of sensors (magnetometer and accelerometer), memory, processor and communication device and can provide information to road users or other systems in vehicles or infrastructure. With the RMU feature, road users can benefit in a variety of ways, like e.g. by alerting a driver for a potentially hazardous situation using the integrated LEDs in the RMUs.

The magnetic sensors of the RMUs detect the vehicles' magnetic field caused by the interaction of two magnetic fields. One is in the vehicle's direction and the other is in the direction of the earth magnetic field. The vehicle magnetic field can be modeled as a magnetic dipole, see Phan [1997]. The change in the magnetic field, as it is observed by a stationary sensor, is a function of the motion of the vehicle and also known as the magnetic signature of the vehicle.

Similarly, a wireless magnetic sensor was proposed by Cheung [2007] to detect the vehicle by putting the magnetic sensor in the middle of a lane. Moreover, vehicle velocity estimation using a pair of magnetic sensors, situated along with the direction of motion was suggested. The estimation method is based on measuring the time difference of the vehicle passing the two sensors represented by detection flags. Having the time and the distance between the sensors determined, the vehicle velocity can easily be calculated. In this scheme different sensor sensitivities can cause inaccurate results. To solve such a problem, a crosscorrelation between readings from two sensors might be used.

Karpis [2012] used this method and determined the velocity by using sampling frequencies, lags of the maximum cross-correlation and the distance between the sensors. Furthermore, the sign of the lags gives an indication of the vehicle heading direction.

Although, vehicle velocity in Cheung [2007] and Karpis [2012] is determined using two magnetic sensors, the vehicle position, which is a very important factor in traffic monitoring, is still uncertain. Therefore, the vehicle trajectory should also be taken into consideration. An Unscented Kalmen Filter technique is proposed by Birsan [2004] to determine vessel position, velocity and magnetic moment based on its magnetic signature. Moreover, in Birsan [2005], such parameters (position, velocity and magnetic moment) are determined using a particle filter assuming multi arrival dimensions and a maneuvering target.

In Wahlström [2010], a non-linear weighted least squares method, $N W L S$, is used for tracking six different vehicle types using readings of two 3-axes magnetic sensors situated on opposite side of the road. The arrangement was the result of an optimization, where the condition number of the Fisher information matrix was minimized.

Finally, It is worthwhile mentioning that in Birsan [2004], Birsan [2005] and Wahlström [2010], the targets are modeled as magnetic dipoles.

The aim of this work is to evaluate a vehicle tracking scheme as a means of monitoring traffic on roads. The scheme can be used as a component in a traffic monitoring system which can provide traffic management system and road maintainers with traffic information. The vehicle 
tracking in this work is achieved by determining vehicle position, velocity and magnetic moment using nonlinear weighted least squares method $(N W L S)$ by means of readings from two 3 -axes magnetic sensors. The sensors are installed adjacently to each other separated by $1 \mathrm{~m}$ distance along the road.

This paper consists of three main sections, The Theory, Simulation and Real life experiments followed by Conclusion. Motion and sensor model are briefly discussed under section Theory. The section Simulation deals with data creation based on different cases of a passing vehicle, $N W L S$ estimation of vehicle states from previously created data and ended with discussion. Finally, The Real life experiments section presents data collection and vehicle states estimation based on real data.

\section{THEORY}

\subsection{Motion Model}

Assume a simple constant velocity vehicle model. The position with respect to the origin $\mathbf{r}_{k}$ is parameterized by three Cartesian components $\mathbf{r}_{k}=\left[r_{k}^{(x)}, r_{k}^{(y)}, r_{k}^{(z)}\right]$ and so is the velocity $\mathbf{v}_{k}=\left[v_{k}^{(x)}, v_{k}^{(y)}, v_{k}^{(z)}\right]$. Such a model can be defined as: Wahlström [2010]

$$
\begin{aligned}
& \mathbf{r}_{k+1}=\mathbf{r}_{k}+T_{s} \mathbf{v}_{k}+\frac{T_{s}^{2}}{2} \mathbf{w}_{k} \\
& \mathbf{v}_{k+1}=\mathbf{v}_{k}+T_{s} \mathbf{w}_{k}
\end{aligned}
$$

where $\mathbf{w}_{k} \sim \mathcal{N}(\mathbf{0}, \mathbf{Q})$ is white Gaussian process noise, $T_{s}$ is the sample time and $k$ is the sampling instance.

\subsection{Sensor Model}

A ferromagnetic bodies (vehicles, vessels,...etc) disturb the uniform magnetic field provided by the earth. Such disturbance can be sensed using magnetic sensors, see Caruso [1999].

The magnetic sensor model is in the form

$$
\mathbf{y}_{k}=\mathbf{h}\left(\mathbf{x}_{k}\right)+\mathbf{e}_{k}
$$

Where the magnetic sensor measures the three Cartesian components of magnetic field $\mathbf{B}$.

For a single magnetic dipole, the measurement equation is

$$
\mathbf{y}_{k}=\mathbf{B}_{0}+\frac{\mu_{0}}{4 \pi} \frac{3\left(\mathbf{r}_{s k} \cdot \mathbf{m}_{k}\right) \mathbf{r}_{s k}-\left|\mathbf{r}_{s k}\right|^{2} \mathbf{m}_{k}}{\left|\mathbf{r}_{s k}\right|^{5}}
$$

where $\mathbf{B}_{0}$ is the stationary earth magnetic field measures by the sensor when there is no passing vehicle, $\mathbf{m}$ is the vehicle magnetic dipole moment, $\mathbf{m}_{k}=\left[m_{k}^{(x)}, m_{k}^{(y)}, m_{k}^{(z)}\right]$ which is at distance $\mathbf{r}_{s}$ away from sensor position, $\mathbf{r}_{s k}=$ $\left[r_{s k}^{(x)}, r_{s k}^{(y)}, r_{s k}^{(z)}\right]$ and $\mu_{0}$ is the permeability of free space, which has a value equal of $4 \pi \times 10^{-7} \mathrm{~F} / \mathrm{m}$.

In Phan [1997] and Wahlström [2010], the vehicle magnetic dipole consists of two components, one related to its reference frame and the other is oriented to the earth magnetic field. The first one representing the permanent magnetization $\mathbf{m}_{0}$ while the second one representing the induced magnetic dipole moment.

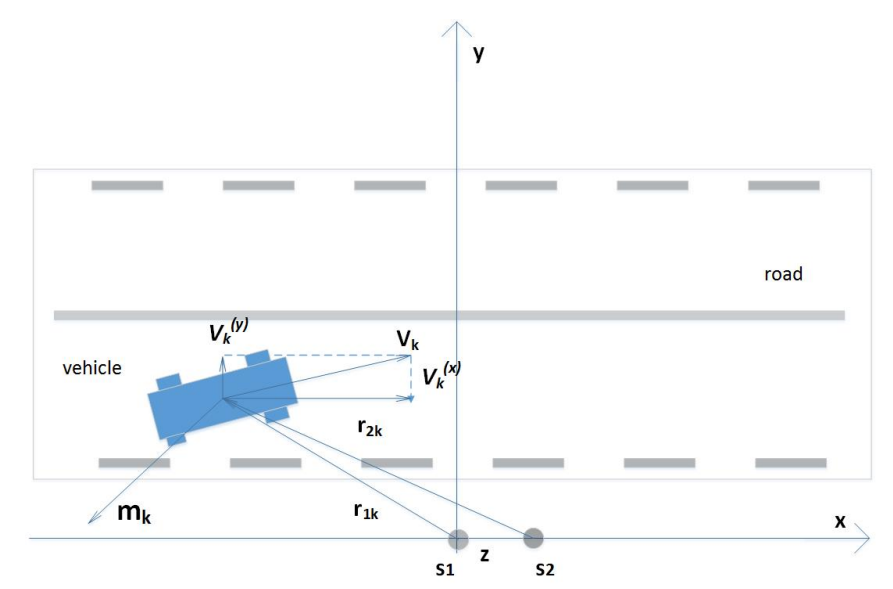

Fig. 1. Vehicle states with respect to the sensors position

The batch model for both process and measurement is given as: Wahlström [2010]

$$
\begin{aligned}
\mathbf{r}_{k} & =\mathbf{r}_{0}+k T_{s} \mathbf{v}_{0} \\
\mathbf{y}_{k} & =\mathbf{B}_{0}+\frac{\mu_{0}}{4 \pi} \frac{3\left(\mathbf{r}_{s k} \cdot \mathbf{m}_{k}\right) \mathbf{r}_{s k}-\left|\mathbf{r}_{s k}\right|^{2} \mathbf{m}_{k}}{\left|\mathbf{r}_{s k}\right|^{5}}+\mathbf{e}_{k}
\end{aligned}
$$

Clearly in Wahlström [2010] the author assumes $\mathbf{w}_{k}=$ 0 in the process model which will produce a constant velocity and magnetic dipole moment. Such an assumption is sensible if the vehicle is assumed to follow a straight line, with constant velocity and without any maneuvers. The vehicle states with respect to the sensors position (S1 and S2) are depicted in Fig.1.

\section{SIMULATION}

Simulation is an important tool for evaluation and validation by which different cases can by assumed and examined. So, before starting the vehicle states estimation (position, magnetic dipole and velocity) based on readings from sensors, data simulating those readings were created. Misalignment of the two sensors was assumed and its effect on the readings of the sensors was simulated and discussed in the Discussion sub-section.

\subsection{Data Generation}

Different cases have been examined. The states in Table 1, using (5) and (6) were used to create the data simulating readings of two sensors when vehicles have magnetic dipole $\mathbf{m}$, initial positions from the origin $\mathbf{r}_{0}$ and velocity $\mathbf{v}$ passing by. The noise is assumed to be white Gaussian noise, see Tönqvist [2006], and covariance matrix $\mathbf{R}$ is assumed to be equal to $10^{-20} \mathbf{I}$ to define the magnetometer noise. The sampling time $\left(T_{s}\right)$ of $0.01 \mathrm{~s}$ was used in the simulation.

The first three cases simulate the same vehicle passing by the two sensors at different velocities while the remaining two cases simulate the same vehicle passed by the two sensors in the same velocity but at different distances away from the sensors. Notice that the first sensor is in the origin and the second is $1 \mathrm{~m}$ away to the right, along the $\mathrm{x}$-axis. 
Table 1. states values were chosen to be simulated

\begin{tabular}{|c|c|c|c|c|c|c|}
\hline Case & Units & 1 & 2 & 3 & 4 & 5 \\
\hline \hline$r^{x}$ & $\mathrm{~m}$ & -5 & -5 & -5 & -5 & -5 \\
\hline$r^{y}$ & $\mathrm{~m}$ & 2 & 2 & 2 & 1.2 & 4 \\
\hline$r^{z}$ & $\mathrm{~m}$ & 0 & 0 & 0 & 0 & 0 \\
\hline$m^{x}$ & $\mathrm{~J} / \mathrm{T}$ & 0.5 & 0.5 & 0.5 & 0.5 & 0.5 \\
\hline$m^{y}$ & $\mathrm{~J} / \mathrm{T}$ & 0.5 & 0.5 & 0.5 & 0.5 & 0.5 \\
\hline$m^{z}$ & $\mathrm{~J} / \mathrm{T}$ & 0.1 & 0.1 & 0.1 & 0.1 & 0.1 \\
\hline$v^{x}$ & $\mathrm{~m} / \mathrm{s}$ & 5 & 15 & 20 & 20 & 20 \\
\hline$v^{y}$ & $\mathrm{~m} / \mathrm{s}$ & 0 & 0 & 0 & 0 & 0 \\
\hline$v^{z}$ & $\mathrm{~m} / \mathrm{s}$ & 0 & 0 & 0 & 0 & 0 \\
\hline
\end{tabular}

The Figures 2, 3 and 4 show the data generated to simulate sensors reading for cases 1,3 and 5 .

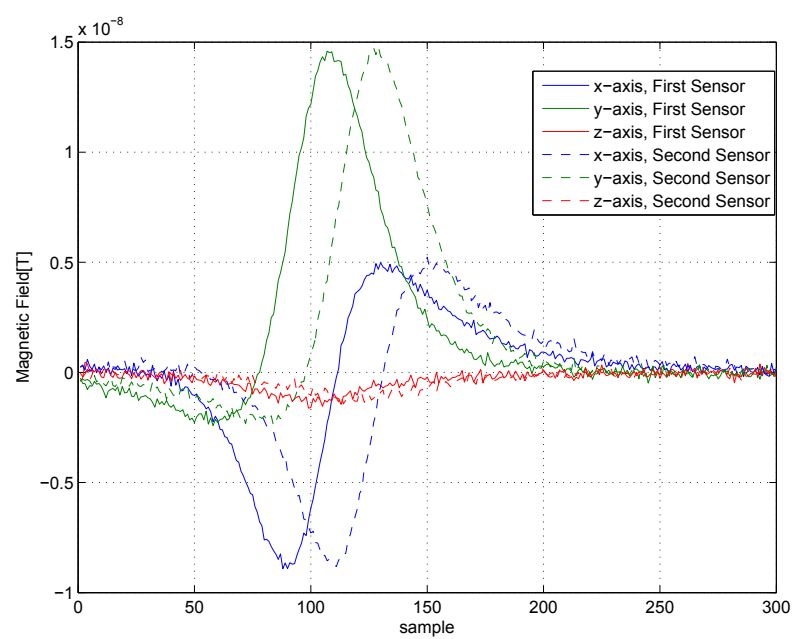

Fig. 2. Readings from the sensors for case 1

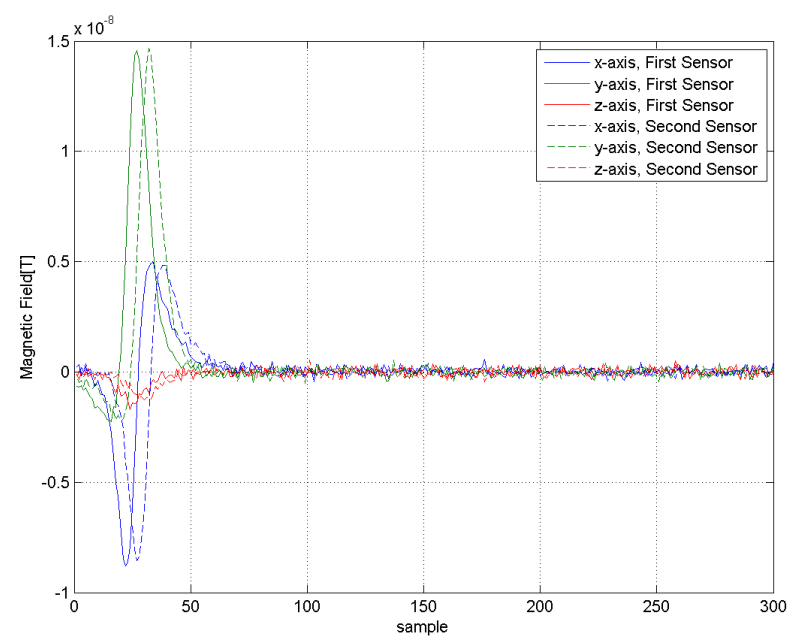

Fig. 3. Readings from the sensors for case 3

We can see that the sketches illustrating the readings of the magnetic sensors are changing. The difference between Fig. 2 and Fig.3 depends on the velocity where the increase in velocity decreases the number of samples, while the difference between Fig.3 and Fig.4 depends on the distance between the vehicle and the sensors, where the large distance results in a weaker signal and a reduced SNR.

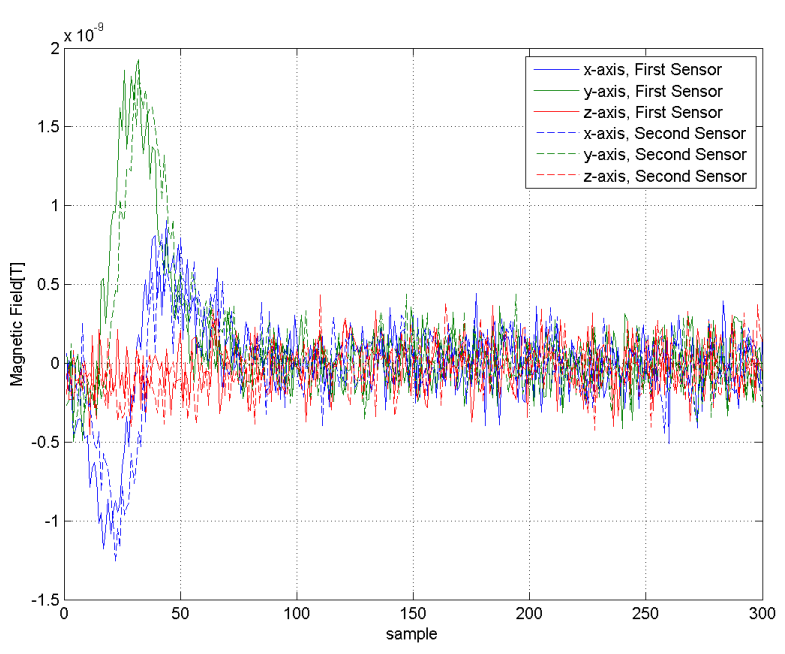

Fig. 4. Readings from the sensors for case 5

\subsection{State Estimation}

The estimation steps mainly depend on finding the solution to the following optimization problem

$$
\begin{aligned}
\hat{\mathbf{x}} & =\operatorname{argmin}_{x} V^{N W L S}(\mathbf{x}) \\
V^{N W L S}(\mathbf{x})= & \sum_{k=1}^{N} \sum_{\text {sensor }=1}^{2}\left(\mathbf{y}_{\text {sensor }, k}-\mathbf{h}_{\text {sensor }, k}(\mathbf{x})\right)^{T} \\
& \mathbf{R}^{-1}\left(\mathbf{y}_{\text {sensor }, k}-\mathbf{h}_{\text {sensor }, k}(\mathbf{x})\right)
\end{aligned}
$$

where $\hat{\mathbf{x}}$ are the estimated stats which minimize the cost function $V(\mathbf{x}), \mathbf{y}_{\text {sensor }}$ is the sensor measurement, $\mathbf{h}_{\text {sensor }}$ is the sensor model in (6) (without $\mathbf{B}_{0}$ ) and $\mathbf{R}$ is the covariance matrix.

The optimization problem was solved using the algorithm propose in Wahlström [2010], Alimi [2009] and Gustafsson [2010] based on nonlinear weighted least squares method $(N W L S)$. The algorithm was initiated using the values given in column ini. The estimation results for the previous cases are presented in the Table 2 .

Table 2. Simulation Results

\begin{tabular}{|c|c|c|c|c|c|c|}
\hline Case & ini. & 1 & 2 & 3 & 4 & 5 \\
\hline \hline$r^{x}$ & -10 & -4.994 & -5.006 & -4.99 & -4.998 & -4.99 \\
\hline$r^{y}$ & 3 & 2.002 & 1.982 & 2.002 & 1.199 & 4.08 \\
\hline$r^{z}$ & 1 & 0.003 & -0.006 & 0.012 & 0.001 & 0.00 \\
\hline$m^{x}$ & 1 & 0.499 & 0.498 & 0.494 & 0.500 & 0.51 \\
\hline$m^{y}$ & 1 & 0.500 & 0.501 & 0.494 & 0.500 & 0.48 \\
\hline$m^{z}$ & 1 & 0.102 & 0.098 & 0.100 & 0.100 & 0.14 \\
\hline$v^{x}$ & 12 & 4.995 & 15.004 & 19.968 & 19.992 & 20.06 \\
\hline$v^{y}$ & 1 & -0.001 & 0.047 & -0.033 & 0.004 & -0.28 \\
\hline$v^{z}$ & 1 & 0.000 & 0.015 & -0.030 & -0.007 & 0.11 \\
\hline
\end{tabular}

\subsection{Discussion}

The estimation results for all cases reveal a very good convergence to the states values used in the data creation. The estimation takes different iteration cycles depending on the case to be estimated. Moreover, in case 5, where the vehicle was far from the sensors and the SNR is low, the estimation algorithm still converges nicely to the states. 
In some cases which are not considered in our simulation cases the estimation algorithm diverges when the vehicle passes very close to the sensors. In these cases we violate a main condition for the magnetic sensor model, which is the distance between the vehicle and the sensors should be greater than the width of the magnetic dipole representing the vehicle itself. In other words, we could not estimate a long vehicle passing close to the sensor using that magnetic sensor model. In that case, we should sub-divide these kind of vehicles into multi-magnetic dipoles, see Wahlström [2010]. In addition, installing the sensors right in the middle of the road would violate a requirement of the magnetic dipole model validity.

There are many articles in which two magnetic sensors are used to detect the vehicle and to determine the velocity easily by using the cross-correlation between records for both sensors and the distance between them, see Karpis [2012]. The maximum cross-correlation of the sensors readings for case 1 occurs at lags 20 , i.e $20 T_{s}$ s. That means, the vehicle which passed the first sensor would pass the second one by that time. Where $T_{s}=0.01$ and $1 \mathrm{~m}$ separates the sensors, vehicle velocity is $5 \mathrm{~m} / \mathrm{s}$. The maximum cross-correlation for case 3 occurs at lags 5 , which means that the vehicle velocity is $20 \mathrm{~m} / \mathrm{s}$. Lastly, the maximum cross-correlation for case 5 occurs at lags 5 . That means the vehicle velocity is $20 \mathrm{~m} / \mathrm{s}$.

Although it is easer to estimate the velocity using crosscorrelation (regardless the errors may occur due to the error in sampling rate and/or the distance between the sensors), we prefer to use $N W L S$ method, as not only the velocity would be determined but also vehicle magnetic moment and position in the $\mathrm{x}, \mathrm{y}$ and $\mathrm{z}$ axes. Vehicle position is a very important factor if we would aim to warn vehicles coming on the other side of a road to avoid a headon collision, or would aim to track vehicle within a sensor network. Besides, we can use both estimation methods in states estimation. A cross-correlation is used as first phase to estimate a good initial velocity guess which can further used in $N W L S$ method as a second phase.

Finally, The sensors setup is very important to assume perfect results. In Wahlström [2010], a calibration of the reference frame is performed using accelerometer and the magnetic sensor as a compass. Fig. 5 and Fig. 6 simulate the readings from the two sensors when yaw $(\Psi)$ and pitch $(\theta)$ angles of the second sensors are changed from 0 to $30^{\circ}$ by a step size of $5^{\circ}$. This change affects $\mathrm{x}$ and $\mathrm{y}$ axes readings in the first case and $\mathrm{x}$ and $\mathrm{z}$ readings in the second one.

Case 1 is simulated again with both yaw and pitch angles equal to $15^{\circ}$. The achieved results, using the same initial values with angles initial values of $0^{\circ}$, are given in Table 3 while the results without considering the angles deviation within the estimation algorithm are depicted in Table 4.

Table 3. Simulation Result

\begin{tabular}{|c|c|c|c|c|c|}
\hline Case & $r^{x}$ & $r^{y}$ & $r^{z}$ & $m^{x}$ & $m^{y}$ \\
\hline 1 & -4.98 & 1.98 & 0.00 & 0.49 & 0.49 \\
\hline \hline$m^{z}$ & $v^{x}$ & $v^{y}$ & $v^{z}$ & $\theta$ & $\Psi$ \\
\hline 0.09 & 4.98 & 0.00 & 0.00 & 14.83 & 14.72 \\
\hline
\end{tabular}

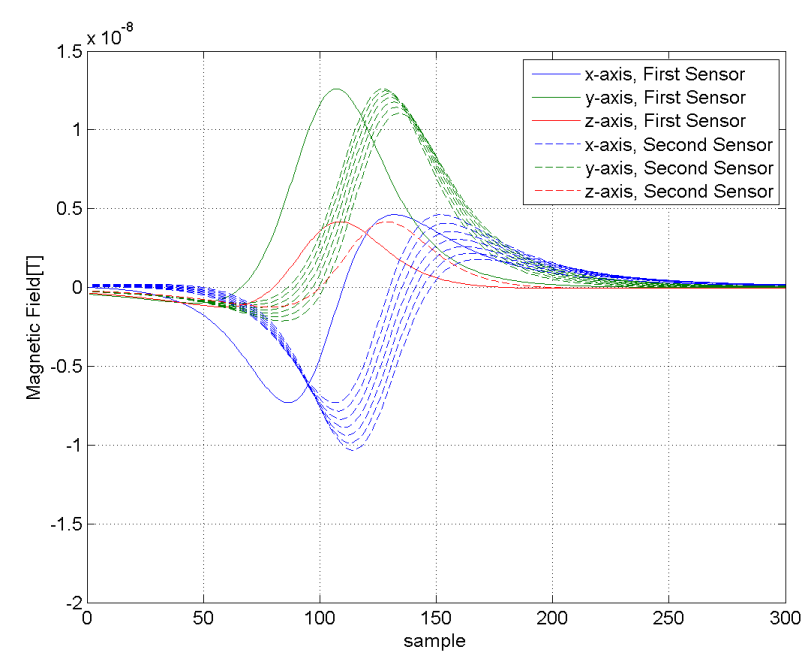

Fig. 5. Changing yaw angle of the second sensor

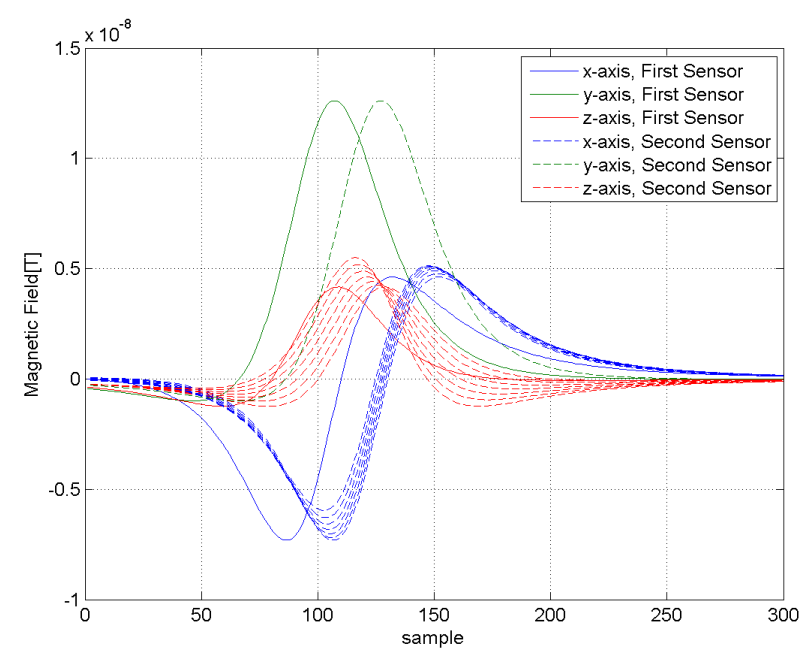

Fig. 6. Changing pitch angle of the second sensor

Table 4. Simulation Result without considering the angles

\begin{tabular}{|c|c|c|c|c|c|}
\hline Case & $r^{x}$ & $r^{y}$ & $r^{z}$ & $m^{x}$ & $m^{y}$ \\
\hline \hline 1 & -4.06 & 1.49 & 0.157 & 0.30 & 0.28 \\
\hline \hline$m^{z}$ & $v^{x}$ & $v^{y}$ & $v^{z}$ & $\theta$ & $\Psi$ \\
\hline 0.002 & 4.079 & 0.15 & -0.18 & - & - \\
\hline
\end{tabular}

The previous tables reveal that the results in the case where the angles deviation effect is ignored are not as accurate as the case where it is considered. In the real experiment, emphasis has been on the accurate alignment of the sensor, thus perfect alignment is assumed as shown in Fig.7.

\section{REAL LIFE EXPERIMENTS}

\subsection{Setup and Data Acquisition}

The wireless sensor nodes for the traffic monitoring system are depicted in Fig. 8. These RMUs are only 7 millimeter high and can be glue attached to the surface. Since they are embedded into the road markings as an additional 


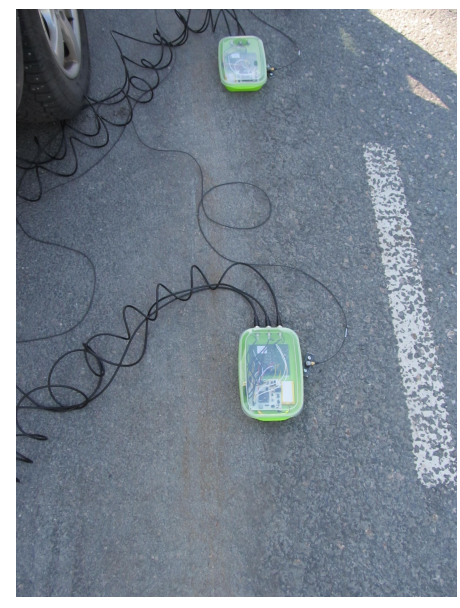

Fig. 7. Collecting data using RMUs prototype

protection, the units sustain snow ploughs and are easily and fast installed on the road surface.

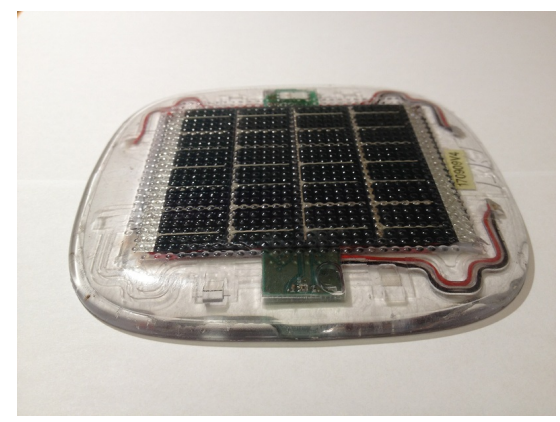

Fig. 8. Integrated RMU, 140mm wide and long and $7 \mathrm{~mm}$ high

A pre-production prototype which is not fully integrated contains a Honeywell 2-axis magnetic sensor type HMC6042 to measure $\mathrm{x}$ and $\mathrm{y}$ axis and 1-axis magnetic sensor type HMC1051 to measure $\mathrm{z}$ axis, were used to collect data from real traffic on a rural road close to LTU, as shown in Fig.7. The 2-axis sensor itself has 225 times amplification, see Honeywell [2007], and then an external amplification was used to amplify 25.5 times more, while the 1 -axis sensors amplified $102 \times 25.5$ times by an external amplification.

Due to a high sampling rate of $16384 \mathrm{~Hz}$ a huge amount of data was collected in four measurement sessions in more than one hour for each.

\subsection{Estimation Of Vehicle States Based on Real Data}

After we applied a modified detection algorithm, see Cheung [2007], to the measurements many signatures for passing vehicles were extracted. The important point is that the two sensors were synchronized with each other, so we applied the detection algorithm on the readings of both sensors at the same time. One of the detection signals for both sensors is shown in Fig.9 which refers to a Volvo SUV passing by.

Table 5 shows the results obtained by applying the discussed estimation algorithm to estimate the states of the

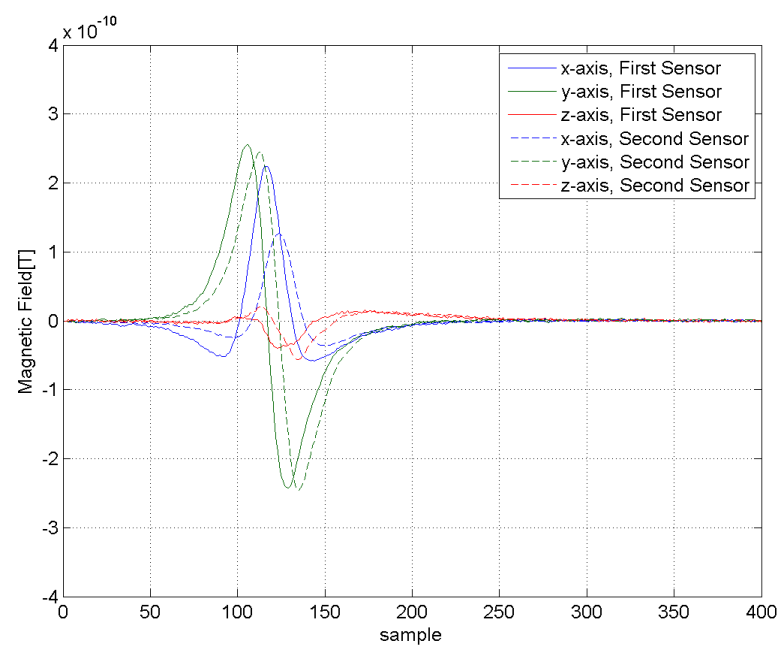

Fig. 9. The Volvo SUV magnetic signature

Volvo SUV. The initial values have been chosen based on our knowledge of sensor sensitivity, road width and speed.

Table 5. Result of states Estimation

\begin{tabular}{|c|c|c|c|c|c|}
\hline case & ini. & Volvo (SUV) & BMW & Toyota & Scania 114L \\
\hline \hline$r^{x}$ & -7 & -15.712 & -13.177 & 16.52 & -21.8 \\
\hline$r^{y}$ & 3 & 2.04 & 0.626 & 3.54 & 8.20 \\
\hline$r^{z}$ & 1 & 1.062 & 1.697 & -6.68 & -6.32 \\
\hline$m^{x}$ & 0.05 & -0.072 & -0.0722 & -0.095 & -1.69 \\
\hline$m^{y}$ & -0.05 & -0.007 & -0.008 & -0.006 & -0.176 \\
\hline$m^{z}$ & 0.05 & 0.0076 & 0.0106 & 0.037 & -0.497 \\
\hline$v^{x}$ & 15 & 22.9 & 18.23 & -24.05 & 18.37 \\
\hline$v^{y}$ & 0 & 1.460 & 2.852 & 7.59 & 1.047 \\
\hline$v^{z}$ & 0 & -1.111 & -1.428 & 7.64 & 5.30 \\
\hline
\end{tabular}
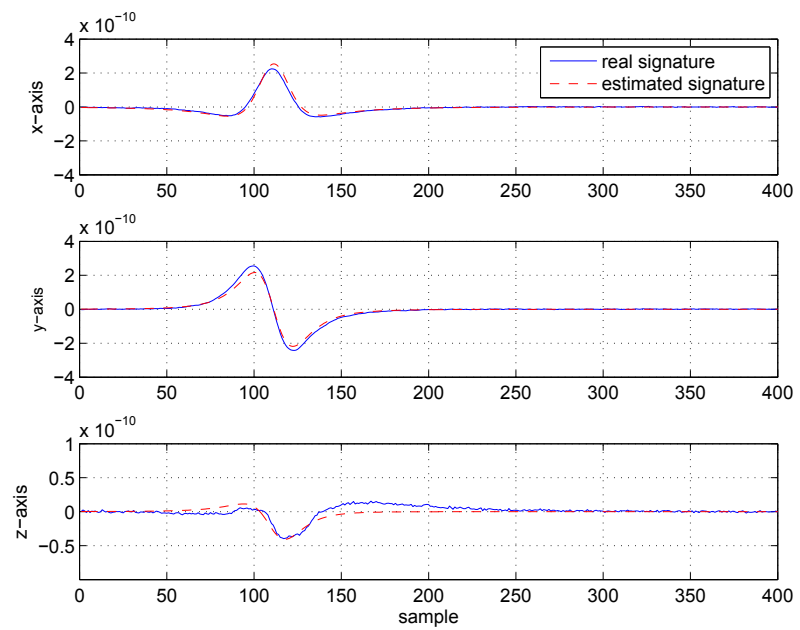

Fig. 10. Estimation comparison of the Volvo case

Fig.10 shows a comparison between the real sensor signals and the reconstructed signals given by substituting the estimation results in $(4)$ (without $\mathbf{B}_{0}$ ). The Figure shows a good fitting between the real and the reconstructed signals.

Table 5 contains three additional estimation cases. The estimation results revealed that the Volvo and the BMW were coming from left to right due to the negative value of $r^{x}$ and the positive value of $v^{x}$. They show also that the BMW was closer to the sensors position than the Volvo, 
Table 6. Standard deviation of Estimated States

\begin{tabular}{|c|c|c|c|c|}
\hline case & Volvo & BMW & Toyota & Scania 114L \\
\hline \hline$r^{x}$ & 0.214 & 0.116 & 0.568 & 0.574 \\
\hline$r^{y}$ & 0.133 & 0.073 & 0.206 & 0.267 \\
\hline$r^{z}$ & 0.155 & 0.089 & 0.268 & 0.213 \\
\hline$m^{x}$ & 0.030 & 0.002 & 0.01 & 0.13 \\
\hline$m^{y}$ & 0.0009 & 0.0006 & 0.0017 & 0.022 \\
\hline$m^{z}$ & 0.0006 & 0.0005 & 0.0041 & 0.039 \\
\hline$v^{x}$ & 0.301 & 0.155 & 0.877 & 0.471 \\
\hline$v^{y}$ & 0.186 & 0.103 & 0.382 & 0.140 \\
\hline$v^{z}$ & 0.220 & 0.122 & 0.362 & 0.177 \\
\hline
\end{tabular}

while the Toyota was coming in the opposite direction due to the positive value of $r^{x}$ and the negative value of $v^{x}$. Moreover, we are almost sure that the Toyota was passing on the other lane because the value of $r^{y}$ is bigger than those of the previous ones. Besides, we can not be very confident about the estimation results for the Scania114L. That was expected as the vehicle was too long compared to distance to the sensors position as we explained earlier. After all, its direction is still able to be recognized (due to $r^{x}$ and $\left.v^{x} \operatorname{sign}\right)$.

The standard deviation of the estimated states of the four cases are depicted in the Table 6. Assume that the noise of magnetometer is white Gaussian, i.e. its samples are i.i.d and are normally distributed with zero mean. As a result $\mathbf{R}$ was used equal to $10^{-22} \mathbf{I} \mathrm{T}^{2}$ which was measured during the target absence after removing the bias caused by earth magnetic field.

\section{CONCLUSIONS}

In order to get safe roads, vehicle tracking can provide substantial information. In this paper, a vehicle tracking scheme was successfully evaluated by determine vehicle position, velocity and magnetic moment. This scheme is achieved by using readings from two 3-axes magnetic sensors. The tracking was performed in both simulation and real life experiments. The real life experiments show a good performance for vehicles on the same side of the road as the installation spot of the sensors, while they give less performance for vehicles on the other lane. Large vehicles create difficulties for the tracking scheme due to an invalidation of single dipole moment model for such vehicles. The velocity and position results gave valuable information about vehicle trajectory while the magnetic moment can be used to reveal vehicle types. The results motivate further and longer tests with a secondary measurement device as ground truth. Test with fully integrated production hardware are now planned and will be conducted in the near future.

\section{ACKNOWLEDGMENT}

Many thanks to my colleagues Khalid Atta and Roland Hostettler for their help and support. Also the effort of Geveko ITS A/S for conducting the experiments and providing the experimental data are gratefully acknowledged.

\section{REFERENCES}

A. Eidehall, J. Pohl, F. Gustafsson and J. Ekmark. "Toward autonomous collision avoidance by steering" Intel- ligent Transportation Systems, IEEE Transactions on, volume. 8, number. 1, pages 84-94, 2007.

W. Birk, E. Osipov and J. Eliasson. "iRoad-cooperative road infrastructure systems for driver support". in Proceedings of the 16th ITS World Congress, 2009.

T. Phan, B. Kwan, and L. Tung. "Magnetoresistors for vehicle detection and identification". in Systems, Man, and Cybernetics, 1997. Computational Cybernetics and Simulation., 1997 IEEE International Conference on, volume. 4. IEEE, 1997, pages 3839-3843.

S. Cheung and P. Varaiya, "Traffic surveillance by wireless sensor networks:Final report". California PATH Program, Institute of Transportation Studies, University of California at Berkeley, 2007.

O. Karpis, "Sensor for vechiles classification" pages 785789, 2012.

M. Birsan, "Non-linear kalman filter for tracking a magnetic dipole" in Proceedings of the International Conference on Marine Electromagnetic (MARELEC04), 2004.

M. Birsan, "Unscented partical filter for tracking a magnetic dipole target" in OCEANS, 2005. Proceedings of MTS/IEEE. IEEE, pages 1656-1659, 2005.

N. Wahlström, J. Callmer, and F.Gustafsson, "Magnetometer for tracking metallic target", in Information Fusion(FUSION), 2010 13th conference on, IEEE, 2010 pages $1-8$.

N. Wahlström, "Target tracking using maxwells equations", p. 87, 2010.

M. Caruso and L. Withanawasam, "Vechile detection and compass application using AMR magnetic sensors", in Sensors Expo Proceedings, Volume 477, 1999.

R. Alimi, N. Geron, E. Weiss and T. Ram-Cohen, "Ferromagnetic mass localization in check point configuration using Levenberg marquardt algorithm", Sensors, Volume 9, number 11, pages 8852-8862, 2009.

D. Törnqvist, statistical fault detection with applications to IMU disturbances", Ph.D dissertation, Linköping, 2006.

F. Gustafsson, "Statiscal sensor fusion", Studentlitterature, 1 edition, 2010.

Honeywell co. "2-axis magnetic sensor circuit HMC6042", 2007. 